\title{
Correction to: Developing a framework to inform scale-up success for population health interventions: a critical interpretive synthesis of the literature
}

Duyen Thi Kim Nguyen ${ }^{1,2,3^{*}}$, Lindsay McLaren ${ }^{4,5}$, Nelly D. Oelke $e^{4,5,6}$ and Lynn Mclntyre ${ }^{4,5}$

\section{Correction to: Global Health Res Policy (2020) 5:18 https://doi.org/10.1186/s41256-020-00141-8}

Following the publication of the original article [1], it was noted that due to a typesetting error the Table 3 layout was incorrect. The correct table is given below.

The original article has been corrected.

\author{
Author details \\ 'Department of Economics, Faculty of Business, University of New Brunswick, \\ 100 Tucker Park Road, P.O. Box 5050, Saint John, New Brunswick E2L 4 L5, \\ Canada. ${ }^{2}$ School of Epidemiology and Public Health, Faculty of Medicine, \\ University of Ottawa, 600 Peter Morand Crescent, Ottawa, Ontario K1G 5Z3, \\ Canada. ${ }^{3}$ New Brunswick Health Research Foundation, 10 Knowledge Park \\ Drive, Fredericton, New Brunswick E3C 2 M7, Canada. ${ }^{4}$ Department of \\ Community Health Sciences, Cumming School of Medicine, University of \\ Calgary, TRW3, 3280 Hospital Dr. NW, Calgary, Alberta T2N 4Z6, Canada. \\ ${ }^{5}$ O'Brien Institute for Public Health, University of Calgary, TRW3, 3280 Hospital \\ Dr. NW, Calgary, Alberta T2N 4Z6, Canada. ${ }^{5}$ School of Nursing, Faculty of \\ Health and Social Development, University of British Columbia, 3333 \\ University Way, Kelowna, British Columbia V1V 1 V7, Canada.
}

Published online: 05 June 2020

\section{Reference}

1. Nguyen, et al. Developing a framework to inform scale-up success for population health interventions: a critical interpretive synthesis of the literature. Global Health Res Policy. 2020;5:18. https://doi.org/10.1186/ s41256-020-00141-8.

The original article can be found online at https://doi.org/10.1186/s41256020-00141-8

* Correspondence: duyen.thi.nguyen@gmail.com

'Department of Economics, Faculty of Business, University of New Brunswick, 100 Tucker Park Road, P.O. Box 5050, Saint John, New Brunswick E2L 4 L5,

Canada

${ }^{2}$ School of Epidemiology and Public Health, Faculty of Medicine, University of Ottawa, 600 Peter Morand Crescent, Ottawa, Ontario K1G 5Z3, Canada Full list of author information is available at the end of the article

(c) The Author(s). 2020 Open Access This article is licensed under a Creative Commons Attribution 4.0 International License, which permits use, sharing, adaptation, distribution and reproduction in any medium or format, as long as you give appropriate credit to the original author(s) and the source, provide a link to the Creative Commons licence, and indicate if changes were made. The images or other third party material in this article are included in the article's Creative Commons licence, unless indicated otherwise in a credit line to the material. If material is not included in the article's Creative Commons licence and your intended use is not permitted by statutory regulation or exceeds the permitted use, you will need to obtain permission directly from the copyright holder. To view a copy of this licence, visit http://creativecommons.org/licenses/by/4.0/. 
Table $\mathbf{3}$ Key phases and actions in the pathway to successfully scaling-up a PHI

\begin{tabular}{ll}
\hline Phase Action & Description \\
\hline Phase 1: Groundwork Preparation & Groundwork phase includes 5 key actions and refers to preparatory actions conducted prior \\
& to implementing scale-up. The primary purpose of Phase 1 is three-fold: i) create a rigorous \\
& and systematic scale-up plan; ii) provide sufficient information for decision-makers to make \\
& an informed decision about whether to implement scale-up; and iii) develop a strong foun- \\
dation for subsequent scale-up phases.
\end{tabular}

Stimulating consideration to scaling-up a PHI To begin the scale-up process, one or more stimulus is required to incites dialogue or action regarding interest to increase the impact of an existing $\mathrm{PHI}$.

Maintaining existing, and building new, stakeholder engagement and buy-in

Human resources are essential to scale-up, and therefore stakeholders must be engaged early and continuously throughout the process. Stakeholders provide the resources, skills, expertise, management, and coordination required to carry out the long and complex scale-up process. Four broad groups of stakeholders were identified: implementers, receivers/ adopters, supporters, and opponents of scale-up.

Conducting/Reviewing assessments

To guide scale-up planning and execution, there are several essential pieces of information that need to be gathered. For example, assessments and data gathered during monitoring and evaluations are pivotal in guiding and informing scale up planning and decisions, such as whether to scale-up, what to scale-up, how to scale-up, where to scale-up, and when to scale-up.

Developing/Retaining/Refining/Modifying resources and stakeholder groups

Throughout the preparatory process there will be actions required to develop, retain, refine, and/or modify various components (i.e., PHI, stakeholders, context, \& capacity) of the scaleup process. For example, with respect to stakeholders, different people or organizations may need to be engaged due to changing roles and responsibilities, changing priorities, competing interests, etc.

Deciding whether to implement scale-up of an Concluding the preparatory phase of scale-up, a decision will need to be made regarding existing PHI whether or not to scale-up the PHI. Deliberations are conducted, typically by a committee of key stakeholders, regarding actions to scale-up a PHI. Many factors go into the decisionmaking process (e.g., evidence of health impacts; stakeholder commitment, costeffectiveness), and the ranked importance of such factors vary between decision makers.

Phase 2: Implementing Scale-Up

Implementing Scale-Up Phase includes 4 key actions. Implementation refers to the process of executing scale-up of the PHI; this phase is only conducted if the PHI is strongly being considered for scale-up. The primary purpose of phase 2 is three-fold: i) successfully implement scale-up; ii) prepare to sustain the scaled-up PHI; and iii) decide how long to sustain the scaled-up PHI.

Continuing / Modifying actions conducted during Ground-work Phase

This action reflects the iterative and dynamic actions of scale-up, and the need to occasionally continue or build-up previous actions. Many previous actions may either be continuing with or without modifications, for example because the focus shifts towards implementing scale-up, unintended consequences, or lessons learned.

Building / Consolidating capacity for scale-up Scaling-up requires many different capacities. Sufficient capacity for scale-up is typically accumulated over time, by way of newly acquiring and/or consolidation. There are various capacities required for scale-up (e.g., PHI design, infrastructure, resources, financial, technical).

Rolling out scale-up implementation strategies Various strategies may be used to implement scale-up of a PHI (e.g., decentralization; integration; replication). Typically, implementation is conducted in a phased or incremental manner over an extended period of time. Occasionally inspections or fines must be enforced to ensure scale-up is being implemented as intended.

Deciding whether to sustain the scaled-up PHI

At some point during the implementation phase, a decision must be made regarding whether the scaled-up PHI should and will be sustained. Sustaining the scaled-up PHI for a longer length of time may not be applicable to all scenarios (e.g., due to the nature of the health issue; availability of resources; changing priorities), and this decision will be unique to the scale-up scenario.

Phase 3: Sustaining the Scaled-Up PHI

Continuing / Modifying previous actions to maintain the scaled-up PHI

Adapting / Evolving to changing components
Sustaining the scaled-up PHI phase includes 2 key actions. Sustaining refers to sustaining the effort to maintain the scaled-up PHI and, thereby sustaining the impact of the scaled-up PHI. The primary purpose of Phase 3 is to successfully sustain the scaled-up PHI for the intended period of time.

This action includes an assortment of previous actions undertaken in the two previous phases. Many previous actions may either be continuing with or without modifications depending on the changing circumstances of the scale-up scenario. The focus shifts from implementing scale-up to maintaining the scaled-up PHI, and due to this shift actions are adjusted accordingly.

To assist in sustaining the scaled-up PHI for an extended length of time, some may need to adapt or evolve their scaled-up PHI based on changes to the key components of scale-up (i.e., context, stakeholders, \& capacity). 